\title{
Global Availability and Use of Over the Counter Antibiotics (OTCA): A Systematic Literature Review
}

\section{Carmen Aceijas $^{1}$ and Dianna Henry Selvaraj ${ }^{2 *}$ \\ ${ }^{1}$ Middlesex University, Faculty of Science and Technology, Department of Natural Sciences, Faculty of Science and Technology London, UK ${ }^{2}$ Chettinad Superspeciality Hospital, Emergency and Trauma Care Department, Kancheepuram, India}

*Corresponding Author: Henry Selvaraj, Chettinad Superspeciality Hospital, Emergency and Trauma Care Department, Kancheepuram, India.

Received: July 17, 2019; Published: August 28, 2019

DOI: $10.31080 /$ ASDS.2019.03.0628

\begin{abstract}
Background: Resistance became widespread from the 1980s and it is estimated that in Europe and US alone, up to 50,000 people die each year due to antibiotic resistant infections. Antibiotics however remain widely misused primarily due to its easy over the counter availability in many parts of the world. Methods: A systematic literature review of the global use and determinants of OTCA updating from the latest systematic literature review in 2009. This review adhered to the PRISMA guidelines for systematic reviews and search strategies were in adherence with Chochrane's MECIR. We reviewed 55 studies on OTCA worldwide published between 2009 and 2017. The worldwide estimate of over the counter antibiotic use and its characteristics were systematically reviewed and described. Results: OTCA use ranged between 20-49\%. Sources of advice were pharmacists, family, or friends [65-75\%]. Penicillin group of antibiotics were the most commonly misused [up to 88\%] and respiratory symptoms were the main reasons for the use even though awareness regarding antibiotic resistance risk or misuse side effects was high [up to 84\%]. Economic factors and previous use with a positive outcome were common reasons for its use. Other determinants such as age, gender, education and socio- economic status were identified but greatly varied across studies' location. Conclusion: This study provides an updated insight into OTCA. Current WHO initiatives should be revitalized, and national efforts set up to curb the problem.
\end{abstract}

Keywords: Over the Counter Antibiotics; Self-Medication; Without Prescription; Anti- Microbials; Antibiotic Resistance

\section{Abbreviations}

ADR: Adverse Drug Reactions; Amox- Amoxicillin; CDDEP: Centre for Disease Dynamics, Economics and Policy; CDRO: Community Drug Retail Outlets; CI: Confidence Interval; CP: Community pharmacies; E-M: Euro-Mediterranean; EU: European Union; FQ: Fluoroquinolones; GI- Gastro Intestinal; IQR- Inter Quartile Range; K, A and E: Knowledge, Attitudes and Experience; K, A and P: Knowledge, Attitudes and Practices; K, B: Knowledge and Beliefs; KSA- Kingdom of Saudi Arabia; NCA: National Competent Authorities; NHS- National Health Services; NP: Non-Prescription; NPA: Non-Prescription Antibiotics; NPA: Non-Prescription Antibiotics; OR: Odds Ratio; OTC: Over the Counter; OTCA: Over the
Counter Antibiotics; Pres: Prescription; SD: Standard Deviation; SM: Self-Medication; SMA: Self-Medication Antibiotics; Uni: University; URTI: Upper Respiratory Tract Infection; UTI: Urinary Tract Infection; WHO: World Health Organisation

\section{Introduction}

Antibiotics are one of the most widely used drugs [1]. However, misuse of these drugs threatens their usefulness [2]. The issue of antibiotic resistance became widespread from the 1980s [2] yet, the over the counter [OTC] antibiotic availability and use continue to be extensive [3]. Currently, it is estimated that in Europe and US alone, up to 50,000 people die each year due to antibiotic resistance infections [4]. 
Antibiotics are legally and illegally sold (OTC) commonly in developing countries and its distribution is poorly regulated [5,6]. This implies that the general public in these countries have access to antibiotics without a prescription or a diagnosis and can hence use them at their own will, promoting over use [7]. Studies suggest that the main reasons for use of over the counter antibiotics [OTCA] in low income countries include high healthcare costs, dissatisfaction with medical practitioners and increased prescription of antibiotics [6]. Use of self-medication is also found to be higher in low and middle-income countries with literacy levels inversely associated to self-medicating [8].

More than half of the antibiotics used around the world are estimated to be from OTCA sales without a prescription [6]. Financial interests from pharmaceutical companies [9] and lack of enforced regulation have been traditionally identified as driving factors of OTCA and to these, the addition of online antibiotic sales adds to the problem [10]. The improper and excessive use of antibiotics is said to be a result of a combination of factors such as physician's practice, knowledge and attitudes of the public regarding antibiotic misuse, self- medication and experience with antibiotics $[11,12]$.

Availability of non-prescribed antibiotics promotes self-medication [13] with the frequent outcome of drug/dosage/indication inappropriateness or unnecessariness [11]. In addition to risks such as failure of treatment, side effects and adverse drug reactions, the rising concern over antibiotic resistance is a strong reminder of the potential dangers antibiotic misuse can pose [13].

Resistance is currently the greatest challenge in infections due to use of resistance to existing antibiotics and the failure to produce any new ones [14]. The 1998 resolution of the World Health Assembly (WHA) urged its Member States to come up with measures to prevent antibiotic misuse following which many countries set up a National Action Plan to address the problem [12].

The previous review in 2009 by Morgan D. J. et al provided evidence that a worldwide problem of OTCA use existed and the safety concerns associated with this. The aim of this review is to update and assess the evidence since the previous systematic review regarding the extent of OTCA use, identify the most commonly used OTCA, pharmacists' attitudes and practices regarding its sales, symptoms commonly used for and its various determinants.

\section{Methods}

This review was adhered to the Preferred Reporting Items for Systematic Reviews and Meta-Analyses (PRISMA) guidelines for systematic reviews [14] and data sources and data search strategies were in adherence with the - Methodological Expectations of Cochrane Intervention Reviews (MECIR).

Primary search was carried out using PubMed, Embase, Google scholar and Medline to identify studies conducted globally between 2009 to 2017 and published in English. The key search terms used were 'Over the Counter', 'OTC', 'Non-Prescription', 'Without Prescription', 'Self Medication', 'Antibiotics', 'Anti-microbials', 'Antituberculosis'. The relevant Medical Subject Heading (MeSH) terms were used along with these to maintain uniformity of terminology across the various databases. Secondary data search included reference lists and bibliographies of the published articles identified in the primary search and grey literature such as conference proceedings, websites and national or government reports.

To provide an updated analysis of the situation from the last comprehensive systematic review [26], studies post 2009 were included. Observational studies such as surveys, mystery shopper reports (in which the patterns of over the counter antibiotic dispensing in pharmacies are explored by an actor taking the role of a client visiting a pharmacy [15] and non-participant observational studies (studies based on observation of actual pharmacist-client interactions at a pharmacy) were included.

Studies conducted in pharmacies, community or university settings and among pharmacists, caretakers, members of the community or among students were included in this review.

Units of analysis were critically appraised using the STROBE checklist and selected for analysis based on evaluation of its quality. They were then grouped separately and data regarding the methodological and outcome variables were extracted. Study results were grouped by Africa, Asia, Middle East, and others (Europe, Australia and America).

The main outcome variable- Weighted non-prescription antibiotic use was estimated from the surveys in pharmacies and calculated both globally and by region following the method used in Morgan et al (2010).

For each region, surveys of OTCA were combined to report frequency of OTCA purchase. To control the different sample sizes in each survey, the percentage of patients sold OTCA was multiplied by the total number of respondents in each survey. The absolute numbers of pharmacy clients who received OTCA for a region were then divided by the total number of survey respondents. 
Other outcome variables included estimate of pharmacy sourced OTCA, most commonly used OTCA, symptoms OTCA were used to treat and characteristics of OTCA use.

Mystery shopper reports and non-participant observational studies were separately grouped and narratively described.

\section{Results}

An initial search identified 469 papers of which 399 were discarded either due to no relevance or failure to fulfil inclusion/ exclusion criteria. The remaining 70 were further screened and 55 studies (42 surveys, 11 mystery shopper reports and 2 non-participant observational studies) were selected for analysis following critical appraisal. 15 studies were excluded due to inappropriate study design, biased methodology or insufficient findings (Figure $1)$.

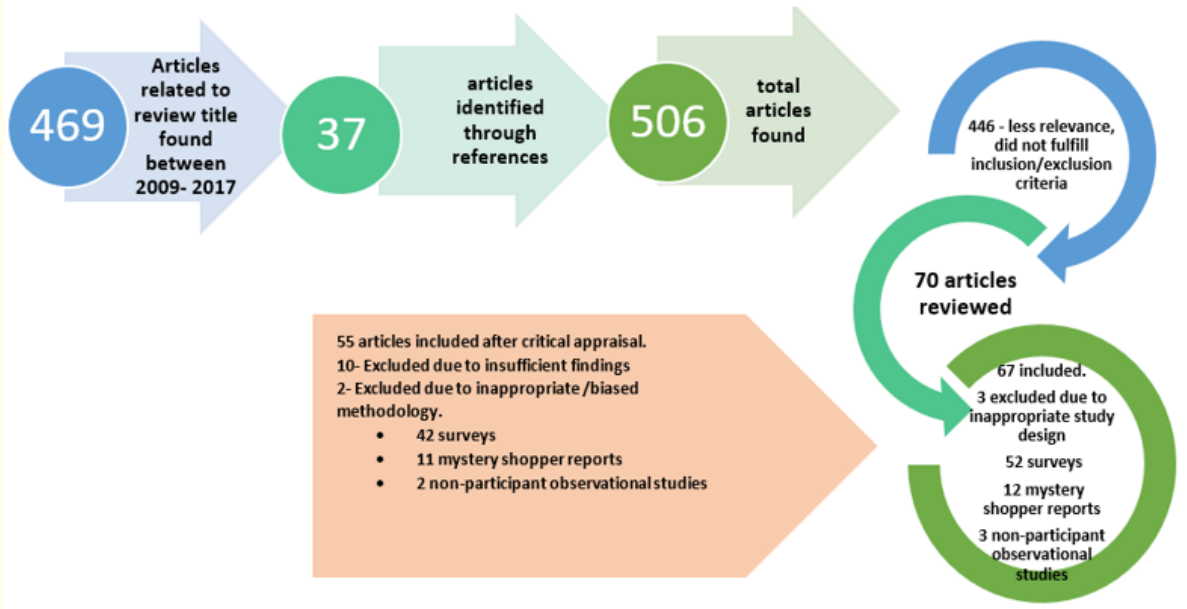

Figure 1: Flowchart of study selection and Region wise distribution of studies.

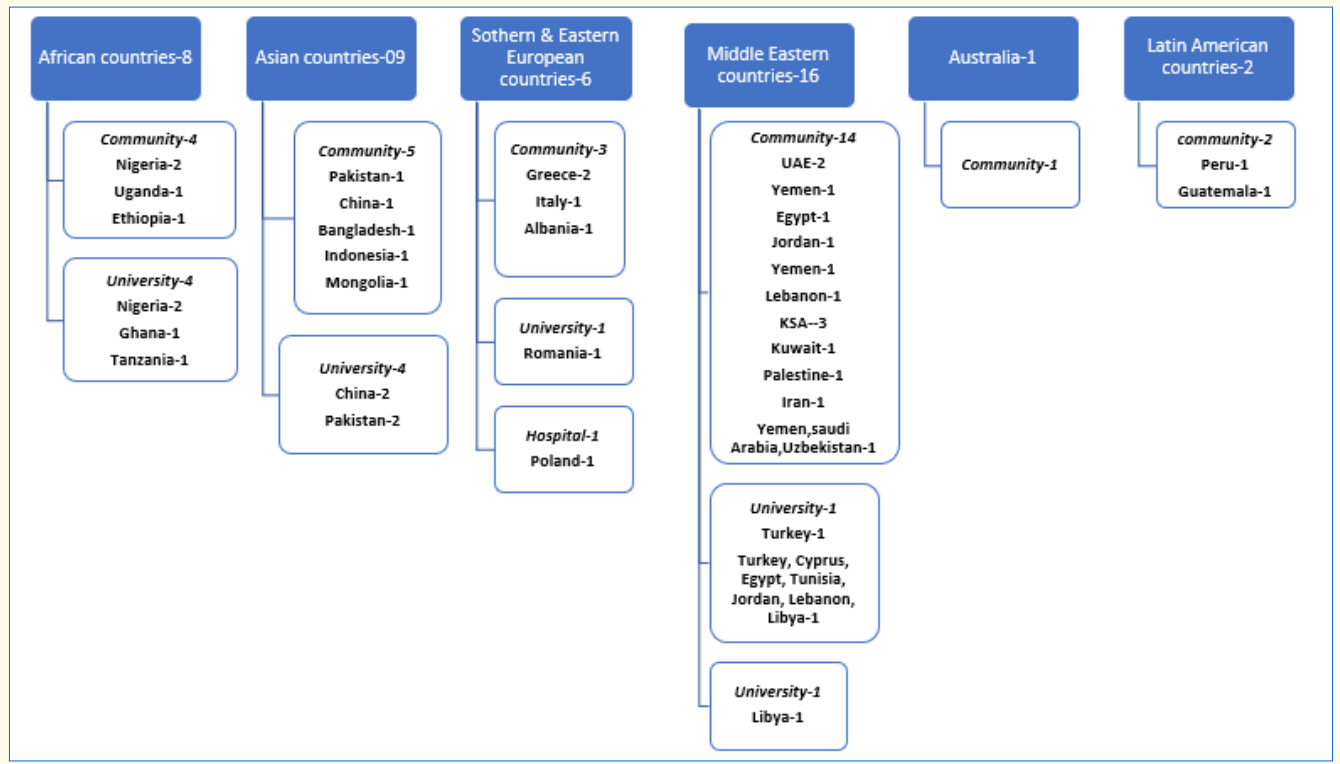

Figure 2: Classification of 42 surveys based on region and setting-Distribution of analyzed surveys depending on the six regions and three settings- Community, hospital and university.

Citation: Carmen Aceijas and Dianna Henry Selvaraj. "Global Availability and Use of Over the Counter Antibiotics (OTCA): A Systematic Literature Review”. Acta Scientific Dental Sciences 3.9 (2019): 92-104. 


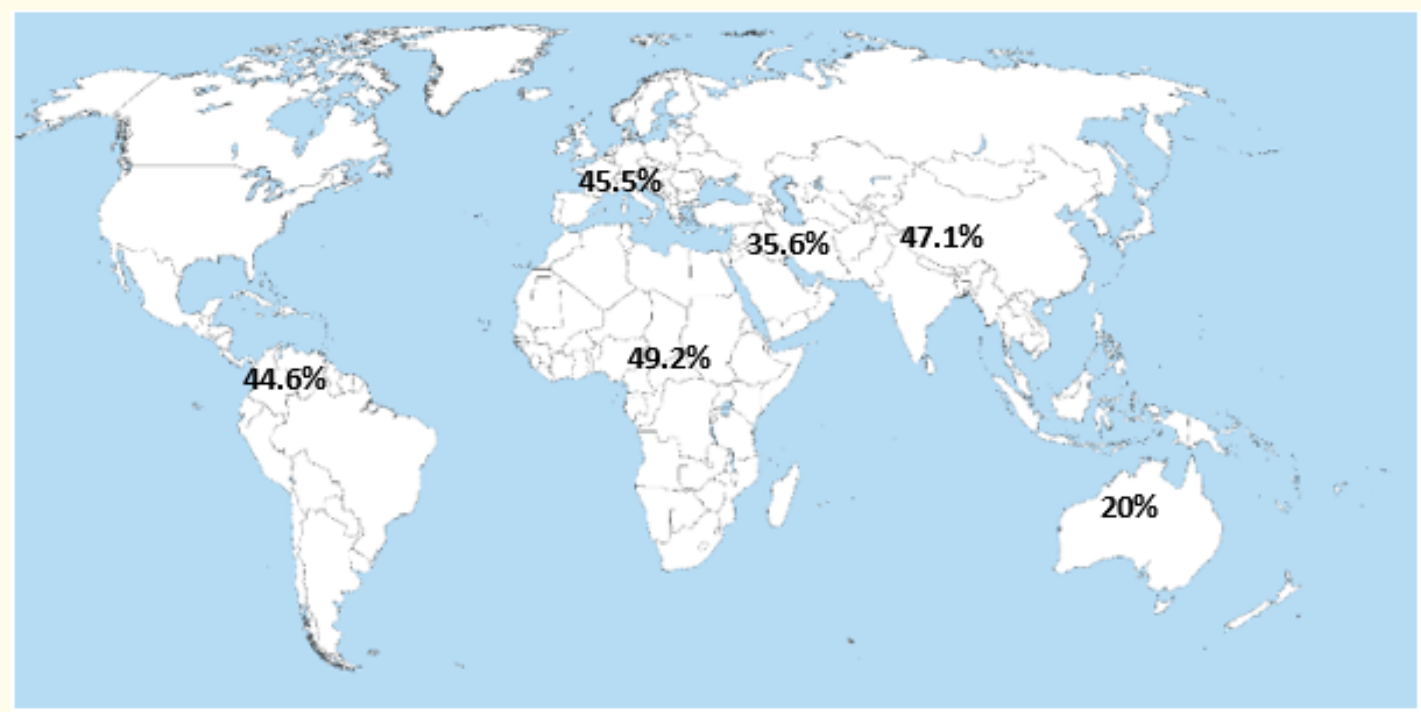

Figure 3: Prevalence of OTCA use.

Percentages of weighted OTCA use are recorded among countries in Africa, Asia, Middle East, Eastern \& Southern Europe, Australia and Latin America.

\section{Worldwide OTCA use and source}

Worldwide OTCA use ranged from $20.2 \%$ to $49.2 \%$ and they were commonly sourced from pharmacies. The regional distribution was:

\section{African countries}

$49.2 \%[2,261 / 4,600]$ of which $48.6 \%$ were sourced from pharmacies with recall periods between 3 months to a lifetime. This estimate includes three papers from Nigeria which determined that OTCA use for menstrual symptoms [24\%] was lowest compared to diarrhoea [46.5\%] and URTI [71.7\%].

The highest OTCA use among these papers was from Uganda [75.70\%]. This study attributes this high use to the increased number of school drop outs, inaccessibility to healthcare due to a weakened healthcare infrastructure following a decade of conflict and long waiting times at the health facilities.

The study from Ghana [70\%] reported OTCA use among tertiary level students. According to this survey, the levels of OTCA use among medically inclined students was found to be lower than those of non-medically inclined students. This paper highlights the importance of educating students about the ill effects of antibiotic misuse. On the contrary, the study among university students in $\mathrm{Ni}$ - geria [56.89\%] reports the higher OTCA use among students in the medical or pharmaceutical faculties due to their presumed knowledge on antibiotics.

The lowest use was in Tanzania [27\%]. This study was conducted among boarding school students averagely aged around 20years. The low OTCA use can be attributed to this study setting in contrast to the other studies

\section{Asian countries}

$47.1 \%[2,631 / 5,588]$ of which $81.05 \%$ were sourced from pharmacies. Recall periods ranged from 1 month to a lifetime.

The highest use [81.25\%] was from a survey in rural areas of Karachi, Pakistan where respondents had a low level of education. Use was much lower in the other two surveys from Pakistan among university students. Among the two, OTCA use among non-medical university students [47.6\%] was marginally lesser than that of nursing students [52.7\%].

Among the surveys from China, OTCA use was highest among parents in rural areas who used OTCA for their children [62\%] compared to use among university students [40.2\% and $47.9 \%$ ]. The lowest use was from Bangladesh [26.69\%], though the OTCA 
sales is prohibited as in many other countries, it is still widely available due to the lack of strict policies and regulations which impose them. This was the case in all the Asian countries analysed.

\section{Southern European countries}

$47.3 \%$ [1093/2,310], Eastern European countries $=42 \%$ [493/1172] with $46.3 \%$ of OTCA in southern and Eastern Europe sourced from pharmacies. Recall periods were 12 months for all surveys from Southern Europe and between 6 months to a lifetime in those from Eastern Europe.

The surveys analysed from Poland [41.4\%] and Macedonia [43.3\%] estimated OTCA use for respiratory infections. All the surveys had similar use rates except Albania [78.14\%]. This study blames the high use of OTCA use in Southern and Eastern Europe to various disparities in the healthcare system such as reimbursement and drug dispensing policies and access to healthcare as compared to Northern and Central Europe.

\section{Middle-East countries}

$35.6 \%$ [4,995/14,029] where $28.14 \%$ were sourced from pharmacies. Recall periods ranged from 1 month to a lifetime. Most of the surveys analysed were from this region and OTCA use varied greatly among them. The survey with the lowest use was from Iran [7.3\%] which was an outlier to this region.

One survey encompassing seven countries namely Cyprus, Egypt, Jordan, Lebanon, Libya, Tunisia and Turkey also had a low OTCA use [19.1\%]. The lowest among these was in Cyprus estimated at $<0.1 \%$ and the highest was in Lebanon-37\%. The low use in Cyprus is reportedly due to the strict regulation of OTCA sales under the EU governance.

The highest estimate was from a survey in a hospital setting in KSA [78.7\%]. The use was however lower in surveys from community settings in KSA [73.7\% and 48\%]. Yemen and Uzbekistan also had a very high use rate [78\% each]. These studies uniformly discuss about the lack of stricter laws to regulate OTCA sales.

\section{Australia}

OTCA use was 20\% among Chinese migrants with a recall period of 12 months. This trend was reported to be more widespread among those migrants who had any inhibitions to using primary healthcare services. Those with a good experience and perception of these services used lesser OTCA.

\section{Latin America}

OTCA use ranged from $33 \%$ in one Peruvian survey to $77-79 \%$ in suburban and city areas in Guatemala, Central America (recall periods 6 and 12 months respectively). The high levels of OTCA use in these countries is reportedly due to OTCA sales being legal and the absence of any law that requires a qualified pharmacist to be present in a pharmacy always.

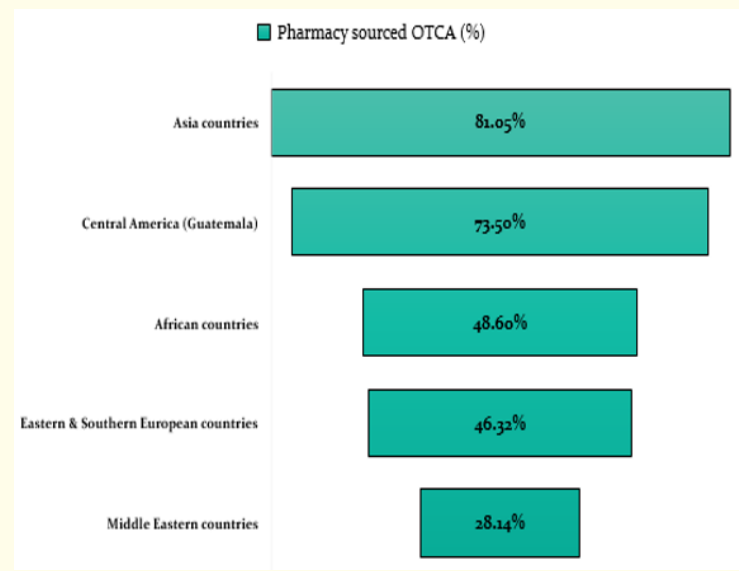

Figure 4: OTCA sourced from pharmacies. The weighted pharmacy sourced OTCA estimates were calculated for the five regions where sufficient data was available.

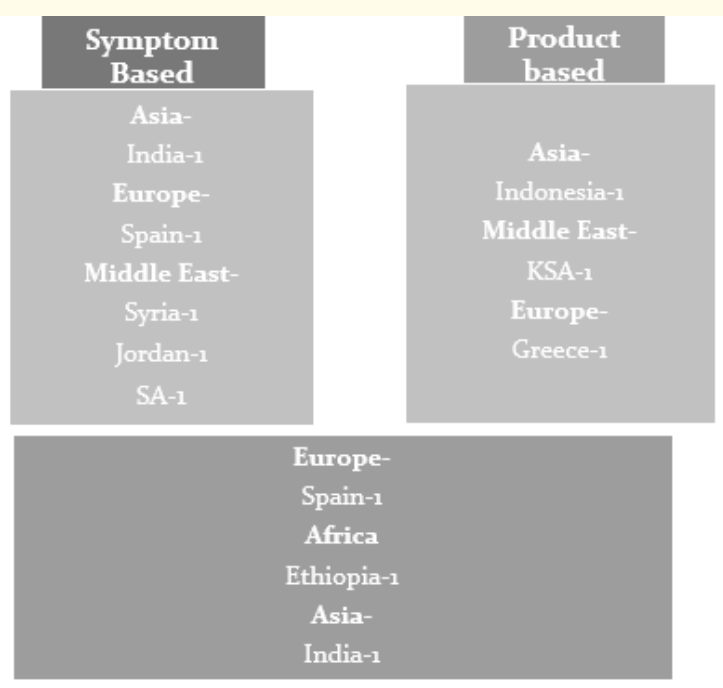

Figure 5: Classification of mystery shopper reports- Scenario and study location-based classification of eleven reports. 


\begin{tabular}{|c|c|c|c|c|c|c|c|c|c|}
\hline Region & Country & $\begin{array}{l}\text { Recall } \\
\text { period }\end{array}$ & $\begin{array}{l}\text { Study } \\
\text { year }\end{array}$ & $\begin{array}{l}\text { Number } \\
\text { of survey } \\
\text { respon- } \\
\text { dents }\end{array}$ & $\begin{array}{c}\text { Total } \\
\text { number } \\
\text { of survey } \\
\text { respon- } \\
\text { dents }\end{array}$ & $\begin{array}{l}\text { Preva- } \\
\text { lence of } \\
\text { OTCA use } \\
\text { (\%) }\end{array}$ & $\begin{array}{l}\text { No. of sur- } \\
\text { vey respon- } \\
\text { dents* } \\
\text { prevalence }\end{array}$ & $\begin{array}{l}\text { Total of no. } \\
\text { of survey } \\
\text { respondents* } \\
\text { prevalence }\end{array}$ & $\begin{array}{c}\text { Weighted } \\
\text { oTCA use } \\
(\%)\end{array}$ \\
\hline \multirow{8}{*}{ Africa } & \multirow{4}{*}{$\begin{array}{l}\text { Nigeria [46] } \\
\text { Lifetime } \\
6 \text { months } \\
\text { Lifetime }\end{array}$} & 3 months & 2008 & 706 & \multirow{8}{*}{$\begin{array}{c}4600 \\
46.5 \% \\
71.70 \% \\
56.89 \%\end{array}$} & $24 \%$ & 16944 & \multirow[t]{8}{*}{226110.17} & \multirow[t]{8}{*}{$49.2 \%$} \\
\hline & & 2012 & 210 & & & 9765 & & & \\
\hline & & 2014 & 403 & & & 28895.1 & & & \\
\hline & & 2009 & 283 & & & 16099.87 & & & \\
\hline & Ethiopia & 12 months & 2014 & 1082 & & $31 \%$ & 33433.8 & & \\
\hline & Ghana & Lifetime & 2008 & 600 & & $70 \%$ & 42000 & & \\
\hline & Uganda & Lifetime & 2012 & 892 & & $75.70 \%$ & 67524.4 & & \\
\hline & Tanzania & Lifetime & 2009 & 424 & & $27 \%$ & 11448 & & \\
\hline \multirow{9}{*}{ Asia } & \multirow{3}{*}{$\begin{array}{l}\text { Pakistan } \\
6 \text { months } \\
\text { Lifetime }\end{array}$} & 6 months & 2015 & 400 & \multirow{9}{*}{$\begin{array}{c}5588 \\
47.60 \% \\
52.70 \% \\
62 \% \\
47.90 \%\end{array}$} & $81.25 \%$ & 32500 & \multirow[t]{9}{*}{263113.8} & \multirow[t]{9}{*}{$47.09 \%$} \\
\hline & & 2014 & 431 & & & 20515.6 & & & \\
\hline & & 2015 & 150 & & & 7905 & & & \\
\hline & Bangladesh & Lifetime & 2013 & 1300 & & $26.69 \%$ & 34697 & & \\
\hline & \multirow{3}{*}{$\begin{array}{c}\text { China } \\
6 \text { months } \\
\text { Lifetime }\end{array}$} & 6 months & 2013 & 731 & & $40.20 \%$ & 29386.2 & & \\
\hline & & 2012 & 854 & & & 52948 & & & \\
\hline & & 2011 & 660 & & & 31614 & & & \\
\hline & Indonesia & 1 month & 2010 & 559 & & $58 \%$ & 32422 & & \\
\hline & Mongolia & 6 months & 2009 & 503 & & $42 \%$ & 21126 & & \\
\hline \multirow{18}{*}{$\begin{array}{l}\text { Middle } \\
\text { East }\end{array}$} & \multirow{2}{*}{$\begin{array}{c}\text { UAE } \\
3 \text { months }\end{array}$} & 12 months & 2009 & 860 & \multirow{8}{*}{$\begin{array}{c}26.40 \% \\
73.70 \% \\
48 \% \\
21.30 \% \\
78 \%\end{array}$} & $39 \%$ & 33540 & & \\
\hline & & 2009 & 1644 & & & 43401.6 & & & \\
\hline & \multirow{4}{*}{$\begin{array}{c}\text { KSA } \\
2 \text { months } \\
3 \text { months } \\
\text { Lifetime }\end{array}$} & 6 months & 2015 & 681 & & $78.70 \%$ & 53594.7 & & \\
\hline & & 2014 & 463 & & & 34123.1 & & & \\
\hline & & 2012 & 400 & & & 19200 & & & \\
\hline & & 2014 & 390 & & & 8307 & & & \\
\hline & \multirow{2}{*}{$\begin{array}{c}\text { Yemen } \\
3 \text { months }\end{array}$} & 4 months & 2013 & 170 & & $25.30 \%$ & 4301 & & \\
\hline & & 2012 & 400 & & & 31200 & & & \\
\hline & Jordan & 3 months & 2006 & 393 & \multirow[t]{10}{*}{14029} & $28.80 \%$ & 11318.4 & \multirow[t]{10}{*}{499522.8} & \multirow[t]{10}{*}{$35.60 \%$} \\
\hline & Lebanon & 4 months & 2011 & 100 & & $32 \%$ & 3200 & & \\
\hline & Palestine & 1 month & 2012 & 400 & & $33 \%$ & 13200 & & \\
\hline & Iran & 1 month & 2012 & 1400 & & $7.30 \%$ & 10220 & & \\
\hline & Libya & 12 months & 2015 & 363 & & $44.50 \%$ & 16153.5 & & \\
\hline & Turkey & 12 months & 2009 & 2696 & & $35.20 \%$ & 94899.2 & & \\
\hline & Kuwait & 12 months & 2014 & 680 & & $27.50 \%$ & 18700 & & \\
\hline & $\begin{array}{l}\text { Cyprus, Egypt, } \\
\text { Jordan, Lebanon, } \\
\text { Libya, Tunisia, } \\
\text { Turkey }\end{array}$ & 12 months & 2009 & 1705 & & $19.10 \%$ & 32565.5 & & \\
\hline & Egypt & 3 months & 2011 & 884 & & $45.70 \%$ & 40398.8 & & \\
\hline & Uzbekistan & 3 months & 2012 & 400 & & $78 \%$ & 31200 & & \\
\hline
\end{tabular}




\begin{tabular}{|c|c|c|c|c|c|c|c|c|c|c|}
\hline \multirow{8}{*}{ Others } & \multirow{2}{*}{$\begin{array}{l}\text { Eastern } \\
\text { Europe }\end{array}$} & Poland & Lifetime & 2011 & 891 & \multirow[t]{2}{*}{1172} & $41.4 \%$ & 36887.4 & \multirow[t]{2}{*}{49251.4} & \multirow[t]{2}{*}{$42.02 \%$} \\
\hline & & $\begin{array}{c}\text { Roma- } \\
\text { nia }\end{array}$ & 6 months & 2011 & 281 & & $44 \%$ & 12364 & & \\
\hline & \multirow{4}{*}{$\begin{array}{l}\text { South- } \\
\text { ern } \\
\text { Europe }\end{array}$} & Greece & 12 months & 2010 & 1139 & \multirow[t]{4}{*}{2310} & $44.6 \%$ & 50799.4 & \multirow[t]{4}{*}{109256.3} & \multirow[t]{4}{*}{$47.30 \%$} \\
\hline & & Italy & 12 months & 2013 & 419 & & $32.7 \%$ & 13701.3 & & \\
\hline & & $\begin{array}{c}\text { Alba- } \\
\text { nia }\end{array}$ & 12 months & 2013 & 350 & & $78.14 \%$ & 27349 & & \\
\hline & & $\begin{array}{l}\text { Mace- } \\
\text { donia }\end{array}$ & 12 months & 2012 & 402 & & $43.30 \%$ & 17406.6 & & \\
\hline & \multirow{2}{*}{$\begin{array}{l}\text { Latin } \\
\text { Amer- } \\
\text { ica }\end{array}$} & Peru & 6 months & 2013 & 1200 & \multirow[t]{2}{*}{1618} & $33 \%$ & 39600 & \multirow[t]{2}{*}{72204} & \multirow[t]{2}{*}{$44.63 \%$} \\
\hline & & $\begin{array}{c}\text { Guate- } \\
\text { mala }\end{array}$ & 12 months & 2014 & 418 & & $78 \%$ & 32604 & & \\
\hline
\end{tabular}

Table 1: Region wise estimation of OTCA use prevalence and pharmacy sourced OTCA.

OTCA use duration, most commonly used OTCA and sources of OTCA advice

Up to $67 \%$ of OTCA users used them for $<3$ days or $>7$ days. The most commonly used antibiotic was Amoxicillin and other Penicillin derivatives $[\leq 88 \%]$. The most common source of OTCA advice was sought from pharmacists $[\leq 75 \%]$ followed by family or friends [ $\leq 65 \%$ ] especially among university students (Table 2 ).
Symptoms OTCA used for, awareness of antibiotic resistance and experience of OTCA adverse effects

OTCA were most commonly used to treat respiratory symptoms which includes cold, cough, sore throat, running nose, upper respiratory tract infection $\left[\leq 84 \%\right.$ ] (Table 3 ). This is despite $3 / 4^{\text {th }}$ of the surveys reporting that $>50 \%$ of the OTCA users were aware of potential adverse effects with a higher awareness identified among university students especially those in medical courses and in urban areas.

\begin{tabular}{|c|c|c|c|c|c|c|c|c|c|}
\hline $\begin{array}{c}\text { Study } \\
\text { Location }\end{array}$ & OTCA Duration & ANTIBIOTIC & RANGE & Source & RANGE & Symptom & RANGE & Reason & RANGE \\
\hline Mongolia & 3-5days-76\% & $\begin{array}{l}\text { Penicillin } \\
\text { (Amoxicillin, } \\
\text { Ampicillin, } \\
\text { Augmentin) }\end{array}$ & $14 \%-88 \%$ & $\begin{array}{c}\text { Pharma- } \\
\text { cist }\end{array}$ & $18 \%-75 \%$ & $\begin{array}{l}\text { Respira- } \\
\text { tory (Cold, } \\
\text { Cough, } \\
\text { Sore throat, } \\
\text { Running } \\
\text { nose, Flu) }\end{array}$ & $7 \%-84 \%$ & $\begin{array}{c}\text { Economic } \\
\text { reasons }\end{array}$ & $6 \%-88 \%$ \\
\hline Nigeria & $\begin{array}{c}\text { 1-4days- } 55.1 \% \\
\text { 5-7days- } 40.8 \% \\
>7 \text { days }-4.1 \%\end{array}$ & Cephalosporin & $2 \%-54 \%$ & $\begin{array}{c}\text { Family/ } \\
\text { Friends } \\
\text { /Neigh- } \\
\text { bours }\end{array}$ & $7 \%-65 \%$ & $\begin{array}{l}\text { Gastroin- } \\
\text { testinal } \\
\text { (Stomach } \\
\text { pain, Diar- } \\
\text { rhoea) }\end{array}$ & $2 \%-84 \%$ & $\begin{array}{l}\text { Time sav- } \\
\text { ing, conve- } \\
\text { nience }\end{array}$ & $17-87 \%$ \\
\hline Nigeria & $\begin{array}{l}<5 \text { days- } 60.7 \% \\
5-7 \text { days- } 85.8 \% \\
>7 \text { days- } 66.7 \%\end{array}$ & Cotrimoxazole & $2 \%-53 \%$ & Media & $2 \%-38 \%$ & $\begin{array}{l}\text { Wounds or } \\
\text { injuries }\end{array}$ & $6 \%-64 \%$ & $\begin{array}{l}\text { Symptoms } \\
\text { simple } \\
\text { enough } \\
\text { to forgo } \\
\text { physician's } \\
\text { visit } \\
\end{array}$ & $\begin{array}{l}27 \%- \\
86 \%\end{array}$ \\
\hline $\begin{array}{l}\text { Saudi } \\
\text { Arabia }\end{array}$ & $\begin{array}{c}2 \text { days-23.8\%, } \\
5 \text { days-38.2\%, } \\
1 \text { week-22.9\%, } \\
1 \text { month-5.6\% }\end{array}$ & Metronidazole & $2 \%-50 \%$ & & & Fever & $4 \%-62 \%$ & $\begin{array}{c}\text { Previous } \\
\text { experience, } \\
\text { positive } \\
\text { outcome }\end{array}$ & $11-82 \%$ \\
\hline
\end{tabular}




\begin{tabular}{|c|c|c|c|c|c|c|c|}
\hline $\begin{array}{l}\text { Saudi } \\
\text { Arabia }\end{array}$ & $1-3$ days $-45.4 \%$ & Tetracycline & $1 \%-28 \%$ & $\begin{array}{l}\text { Urinary } \\
\text { tract infec- } \\
\text { tions }\end{array}$ & $7 \%-42 \%$ & $\begin{array}{l}\text { Easy avail- } \\
\text { ability of } \\
\text { OTC antibi- } \\
\text { otics }\end{array}$ & $\begin{array}{l}29 \%- \\
72 \%\end{array}$ \\
\hline \multirow[t]{4}{*}{ Libya } & $\begin{array}{c}\text { Less than } 3 \\
\text { days- } 15 \%\end{array}$ & $\begin{array}{l}\text { Macrolides } \\
\text { (Erythromy- } \\
\text { cin, Azithro- } \\
\text { mycin) }\end{array}$ & $3 \%-21 \%$ & Pain & $\begin{array}{l}12 \%- \\
40 \%\end{array}$ & $\begin{array}{l}\text { Previously } \\
\text { prescribed } \\
\text { antibiotics } \\
\text { by a doctor } \\
\text { for similar } \\
\text { symptoms }\end{array}$ & $11-64 \%$ \\
\hline & & Ciprofloxacin & $9 \%-15 \%$ & $\begin{array}{c}\text { Dental } \\
\text { (Tooth } \\
\text { pain) }\end{array}$ & $1 \%-20 \%$ & $\begin{array}{l}\text { Inacces- } \\
\text { sibility to } \\
\text { healthcare } \\
\text { services }\end{array}$ & $1 \%-35 \%$ \\
\hline & & & & Allergies & $3 \%-15 \%$ & $\begin{array}{c}\text { Lack of } \\
\text { trust on } \\
\text { physicians } \\
\text { or avoiding } \\
\text { the physi- } \\
\text { cian }\end{array}$ & $5-30 \%$ \\
\hline & & & & & & $\begin{array}{l}\text { Left over } \\
\text { antibiotics } \\
\text { from previ- } \\
\text { ous pre- } \\
\text { scriptions, } \\
\text { Afraid to } \\
\text { go to the } \\
\text { physician }\end{array}$ & $16 \%, 2 \%$ \\
\hline
\end{tabular}

Table 2: OTCA duration-Estimates from six surveys; Most commonly used OTCA-Estimates from twenty surveys; Source of OTCA information- Estimates from 22 surveys; Symptoms OTCA used for-Estimates from 18 surveys, Reasons for OTCA use- Estimates from twenty-three surveys.

\begin{tabular}{|c|c|c|c|c|c|c|}
\hline Country (Year conducted) & Ref. no. & $\begin{array}{c}\text { Respiratory } \\
\text { symptoms }\end{array}$ & Sinusitis & $\begin{array}{c}\text { Urinary tract } \\
\text { infection }\end{array}$ & $\begin{array}{c}\text { Gastro-intestinal } \\
\text { symptoms }\end{array}$ & $\begin{array}{c}\text { Otitis media/ } \\
\text { ear pain }\end{array}$ \\
\hline \multicolumn{7}{|l|}{ Africa } \\
\hline Ethiopia (2015) & 5 & $75.09 \%$ & -- & -- & -- & -- \\
\hline \multicolumn{7}{|l|}{ Europe } \\
\hline Catalonia, Spain (2008) & 7 & $51.70 \%$ & -- & $79.70 \%$ & -- & -- \\
\hline Gipuzkoa, Spain (2009) & 29 & -- & -- & $18.70 \%$ & -- & -- \\
\hline \multicolumn{7}{|l|}{ Middle East } \\
\hline Syria (2008) & 10 & -- & $97 \%$ & -- & -- & -- \\
\hline Jordan (2015) & 16 & $97.60 \%$ & $48.50 \%$ & $83.30 \%$ & $83 \%$ & $83.30 \%$ \\
\hline Saudi Arabia (2011) & 25 & $86 \%$ & $57 \%$ & $27 \%$ & $35 \%$ & $44 \%$ \\
\hline \multicolumn{7}{|l|}{ Asia } \\
\hline Andhra Pradesh, India (2016) & 12 & $51.25 \%$ & -- & -- & -- & -- \\
\hline Bangalore, India (2013-'14) & 13 & $71.30 \%$ & -- & -- & $63 \%$ & -- \\
\hline
\end{tabular}

Table 3: Rates of OTCA dispensing- Based on study location and symptom presented. 


\begin{tabular}{|c|c|}
\hline Symptom & Commonly dispensed antibiotics \\
\hline $\begin{array}{l}\text { Respiratory symp- } \\
\text { toms }\end{array}$ & $\begin{array}{l}\text { Penicillin } \\
\text { Amoxicillin- 8\%-100\% } \\
\text { Amoxicillin+Clavulanic acid- 8\%-100\% } \\
\text { Macrolide } \\
\text { Azithromycin- 15\%-50\% } \\
\text { Cephalosporin- 3\%-10\% }\end{array}$ \\
\hline $\begin{array}{l}\text { Urinary tract } \\
\text { infection }\end{array}$ & $\begin{array}{l}\text { Fluoroquinolones-15\%-80\% } \\
\text { Cephalosporin- } 2 \%-6 \%\end{array}$ \\
\hline $\begin{array}{l}\text { Gastro intestinal } \\
\text { symptoms }\end{array}$ & Fluoroquinolones- 2\%-66\% \\
\hline Otitis media & $\begin{array}{l}\text { Penicillin- } 15 \%-44 \% \\
\text { Macrolide- } 4 \%-20 \%\end{array}$ \\
\hline
\end{tabular}

Table 4: Commonly dispensed OTCA.

Symptoms were grouped into Respiratory symptoms, UTIs, GI symptoms and Otitis media

First-hand experience of adverse effects was detected among 7-25\% of OTCA users of which Gastrointestinal symptoms (e.g.: diarrhoea) and allergies were the most commonly reported adverse effects that were experienced.

\section{Determinants for 0TCA use}

Economic reasons were the most common reason for use (Table 2). Socio economic variations and determinants leading to OTCA use varied widely across regions. Overall, determinants found associated with an increased use of OTCA were: Gender [Male], Education [Higher educational status] and socio-economic status [Lower socio- economic status] and Age [Being 40 years or older]. Use of OTCA for children increased with the child's age and with the number of children in the family.

Mystery shopper reports and Non-Participant observation studies A total of 13 reports were analysed (Figure 5).

The most commonly dispensed antibiotics for respiratory symptoms were Penicillin group antibiotics mainly Amoxicillin [8-100\%] and amoxicillin-clavulanic acid combination [8-100\%] (Tables 3 and 4). Dispensing was significantly influenced by smaller size of the pharmacy [9]. In opposition, pharmacists who had attended more antibiotic awareness campaigns and women had lower dispensing rates [10].
Not all pharmacists ( $\leq 75 \%)$ asked additional information such as drug allergies or presence of other symptoms before dispensing OTCA while 43-95\% gave advices on dose or duration to the clients purchasing OTCA.

18-55\% pharmacists refused to dispense OTC commonly citing administrative reasons or concerns over health of the client and $2-47 \%$ advised clients to visit the physician.

Penicillin was the most commonly requested OTCA amongst which amoxicillin and clavulanic acid was the most common. Almost as high as $100 \%$ of requests were dispensed. Upto $53 \%$ of clients were asked additional questions on the intended OTCA use or advised on appropriate usage. Main reasons for dispensing OTCA were that other pharmacies would dispense even if they refused and the lack of a defined OTCA list [11]. The main reason for refusal was the need for a prescription or the unavailability of the requested antibiotic.

68-90\% of interactions in pharmacies recorded during non-participant observational studies involved OTCA. The most commonly dispensed OTCA were penicillin [ $\leq 62 \%$ ]. Rural pharmacies were reported to be dispensing older antibiotics such as clotrimoxazole and chloramphenicol more frequently [16]. Respiratory symptoms were the most commonly presented $[\leq 77 \%]$.

\section{Discussion}

This review estimates the extent of OTCA use around the world ranging from $20-49 \%$. Two thirds of the regions analysed in this review estimate that almost half of their population use OTCA, the highest of which was in African countries estimated at 49\%. However, in comparison to the estimates of the global non-prescription antibiotic use in 2009 by Morgan et al, this trend apparently has reduced in countries from Africa, Asia and the Middle East and increased in countries from Southern and Eastern Europe and Latin America. Reduction in OTCA use can be attributed to widespread efforts by authorities. The WHO in 2000 issued a report on overcoming antimicrobial resistance containing strategies and policies to be followed globally to promote the judicious use of antimicrobials and making them 'Widely but wisely' used. Though self-medication was reported useful in resource limited settings, it stressed upon the importance of responsible advertising, campaigns and measures to educate the public and reduce bad practices in selfmedication $[17,18]$. 
Other efforts include 'Global Antibiotic Resistance Partnership' GARP established among countries in Asia and Africa in 2009, 'Chennai declaration' in 2012 and measures taken by regional authorities [19-21]. Globally, over 60 antibiotic awareness campaigns were identified out of which 63\% were conducted as part of WHO's annual 'World Antibiotic Awareness Week [10]. Almost all these campaigns that were evaluated formally post implementation and execution proved to have a positive impact in reducing inappropriate antibiotic usage [22].

In Southern and Eastern Europe, despite OTCA being illegal, use was still high owing to multiple factors such as high healthcare costs, inaccessibility, and storage of antibiotics at home stressing the need for reinforcement of the policies and laws in these regions $[23,24]$.

Globally, non-prescription antibiotics were largely sourced from pharmacies $[25,26]$. However, comparison of current pharmacy sourced OTCA estimates with those in 2009 by Morgan et al show a decline while the use of other sources has increased. Several studies report its availability from the internet, storage of left over antibiotics from previous prescriptions, friends or relatives, primary health centres, public health facilities or medical stores [27-29]. Asian and American studies report OTCA availability in corner stores, super markets or veterinary shops [30-32]. Black markets were also reported sources in regions such as America where they are sometimes manufactured and smuggled from regions where OTC sales laws are not very strict [33].

Studies from the Middle East report the purchase of antibiotics from abroad pharmacies $[34,35]$. This can be due to the sheer number of expatriates living in this region from countries where OTCA can be easily obtained from pharmacies [36]. A person's eligibility to benefits from medical insurance in these countries also was a predictor to this practice [34].

A wide range of socio-economic variabilities in OTCA use were reported from each region. The major statistically significant predictors being gender, socio-economic status, education levels and age $[37,38]$. Small pharmacies and pharmacists in low income areas were found to be dispensing more OTCA especially the expensive ones to increase income $[39,40]$.

The most commonly used OTCA are penicillin group of antibiotics especially amoxicillin. This could be driven by relatively low cost and broad-spectrum activity of amoxicillin as compared to other antibiotics [41]. Overuse of these antibiotics as mentioned earlier is a driving force for emergence of resistance [42]. According to WHO's global report on surveillance of antimicrobial resistance in 2016, non-penicillin susceptible microbial chains have been identified in all WHO regions. Additionally, penicillin and other beta lactam antibiotics are one of the most common drugs to cause allergies ranging from skin rashes to angioedema and anaphylaxis in severe cases [43]. Awareness of these adverse effects and knowledge on safe antibiotic use practices and antibiotic resistance was high among the urban population and among university students. However, misuse was still widely prevalent.

OTCA were commonly used for upper respiratory tract symptoms such as sore throat, cough or common cold. However, this practice is highly unwarranted as these symptoms are of viral aetiology in more than $50 \%$ of the cases and antibiotics have no effect on these viruses $[11,44]$. Antibiotics are also not usually necessary for sinusitis, diarrhoea, fever due to viral infections, uninfected wounds, menstrual symptoms or pain [43].

Several inappropriate practices pertaining to its dose and duration, storage of leftover antibiotics or sharing of antibiotics with friends and relatives are linked to OTCA use [3,45]. Duration of antibiotic treatment is highly individualized based on factors such as a person's immune status and the microbial agent involved and must be done by a qualified professional $[46,47]$. Incorrect duration of treatment ranging from less than 3 days to more than a month leads to antimicrobial selection pressure [8]. Also, frequent exposure of the GI commensals to antibiotics in incorrect doses may cause UTIs in women.

Advice regarding OTCA use was sought commonly from pharmacists who are often seen as the 'First point of call [17]. Pharmacists however rarely questioned clients on their allergies or informed them about possible side effects to dispensed OTCA [26]. Other OTCA dispensing practices were also unsatisfactory such as unnecessary dispensing of antibiotics, not advising users on information such as dose and duration of antibiotics dispensed and not referring clients to a physician when needed. Pharmacists should be aware of their role in ensuring proper use of antibiotics in the community and should responsibly advice clients on its use [5]. 
Some of the most common determinants of OTCA use include reduced affordability or accessibility of healthcare, previous experience with the same antibiotic such as a doctor's prescription of the antibiotic for similar symptoms, easy availability or lack of time [23]. These determinants varied depending on the economic status of the region.

In addition to weak and poorly enforced regulations limiting OTCA sales, several other factors are identified as mentioned. Regional authorities are responsible for organising committees by bringing together various stakeholders such as policy makers, healthcare workers and community members to identify and address these determinants effectively. Pharmacists should be more vigilant, and measures should be taken to increase knowledge and awareness among them so that they can promote good practice under their domain. Regular surveillance of antibiotic sales, use and resistance should be standardised protocols to keep track of these parameters and monitor them so that the planning of interventions will be convenient and efficacious.

Antibiotic availability is essential especially in regions where healthcare resources are unaffordable or inaccessible. In such areas, mortalities or morbidities arising from unavailability of antibiotics or failure of timely treatment using antibiotics pose a bigger concern than antibiotic resistance. Authorities should therefore cautiously implement new strategies and review existing ones to regulate OTCA use. Widespread campaigning to improve awareness and knowledge among the general community as well as among healthcare workers has a proven success rate and should be carried out in addition to other measures implemented.

Storage of prescribed antibiotics from not having finished the treatment duration and use of old prescriptions to purchase antibiotics should be monitored and people should be educated on its ill effects. Excess antibiotics should be asked to be returned to pharmacies.

This study has a few limitations which include the analysis of studies with varied methodologies, recall periods, target populations, demographic and socio-economic variations and time frames. Factors such as seasonal variations, cultural variations and GDPs can all affect OTCA use behaviour and act as confounding factors among the studies due to which data obtained and analysed may not be entirely the same as the current trends in those regions. Some relevant studies could have been missed out during data search from certain regions affecting the overall results. The results are subject to a language bias arising from the selection of papers published only in English language.

Overall, this systematic review is an indication that future research needs to be done to accurately estimate OTCA use, determinants and characteristics using a standardized study design and analysis, implemented and executed uniformly in regions around the world. This needs to be done so that appropriate region-specific policies, rules and regulations can be introduced by the authorities and relevant measures can be taken to deal with OTCA misuse and monitor antibiotic resistance.

\section{Key Points and Conclusion}

1. Penicillin was the most commonly misused antibiotic and OTCA were most commonly used to treat respiratory symptoms.

2. Even in countries where OTCA laws are present, obtaining these drugs through illegal sources such as veterinary shops, trafficking from countries where such laws are not present, online sources etc. are common.

3. Pharmacists played a huge role in antibiotic misuse due to incorrect and inefficient dispensing practices.

4. The percentage of pharmacy sourced antibiotics have decreased and auxiliary sources such as leftover antibiotics, antibiotics sourced from friends or relatives, online sources etc. have increased highlighting the importance of introducing more widespread awareness against antibiotic misuse.

5. Regular surveillance of antibiotic sales use and resistance should be adopted worldwide and the importance of OTC availability of antibiotics in countries without easy access to healthcare should be kept in mind while imposing rules and regulations regarding OTCA.

\section{Sources of Support}

This study received no funding by either public or private organisations or sponsors.

\section{Ethical Considerations}

Ethical approval was obtained from the NSS ethics committee, School of Science and Technology, Middlesex university [NSESC Ref No: 2276]. 


\section{Acknowledgements}

A massive and heartfelt thank you to all my colleagues at Middlesex University and my family without whom this research would not have been possible.

\section{Bibliography}

1. National Health Services - NHS. "Antibiotics" (2016).

2. Wayne W LaMorte. 'The Evolution of Antimicrobial Resistance" (2016).

3. WHO. "Survey of Non-Prescribed Use of Antibiotics for Children in An Urban Community in Mongolia" (2010).

4. O’Neill J. “Review on antimicrobial resistance” (2014).

5. Centers for Disease Control-CDC. "Facts About Antibiotic Resistance" (2016).

6. Togoobaatar G., et al. "Survey of non-prescribed use of antibiotics for children in an urban community in Mongolia". Bulletin of the World Health Organization 88 (2010): 930-936.

7. WHO- Regional Office for Europe. "Pharmacists Have Decisive Role in Combating Antibiotic Resistance, Says New WHO European Survey" (2014).

8. Shah S., et al. "Self-medication with antibiotics among nonmedical university students of Karachi: a cross-sectional study". BMC Pharmacology and Toxicology 15 (2014).

9. M J Wood. Over-the-counter antibiotics". Journal of Antimicrobial Chemotherapy 44 (1999): 149-150.

10. Ventola LC. "The Antibiotic Resistance Crisis: Part1: Cause and Threats". Pharmacy and Therapeutics 40 (2015): 277-283.

11. Grigoryan, L., et al. "Self-medication with Antimicrobial Drugs in Europe”. Emerging Infectious Diseases 12 (2016): 452-459.

12. Fadare JO and Tamuno I. "Antibiotic self-medication among university medical undergraduates in Northern Nigeria". Journal of Public Health Epidemiology 3 (2011): 217-220.

13. Shah S., et al. "Self-medication with antibiotics among nonmedical university students of Karachi: a cross-sectional study". BMC Pharmacology and Toxicology 15 (2014).
14. WHO. "Antibiotic Resistance Factsheet" (2016).

15. Butler MS and Buss AD. "Natural products - the future scaffolds for novel antibiotics?" Biochemistry and Pharmacology 71 (2006): 919-929.

16. Cars $O$ and Nordberg P. "Antibiotic resistance -The faceless threat". International Journal of Risk and Safety in Medicine 17 (2015): 103-110.

17. Lee CR., et al. "Strategies to Minimize Antibiotic Resistance". International Journal of Environmental Research and Public Health 10 (2013): 4274-4305.

18. Saam M., et al. "Evaluation of antibiotic awareness campaigns". [eBook] Geneva: World Health Organization (2016).

19. Ocan M., et al. "Household antimicrobial self-medication: a systematic review and meta-analysis of the burden, risk factors and outcomes in developing countries". BMC Public Health 15 (2015): 742.

20. Wayne W LaMorte. 'The Evolution of Antimicrobial Resistance" (2016).

21. WHO. "Survey of Non-Prescribed Use of Antibiotics for Children in An Urban Community in Mongolia" (2010).

22. PRISMA statement (2015).

23. Cochrane. Cochrane Library (2017).

24. Nga D., et al. "Antibiotic sales in rural and urban pharmacies in northern Vietnam: an observational study". BMC Pharmacology and Toxicology 15 (2014).

25. Akinyandenu Olusegun and Adeniyi Akinyandenu. "Irrational Use and Non - Prescription Sale of Antibiotics in Nigeria: A Need for Change". JSIR journal 3 (2014): 251-257.

26. Gebeyehu E., et al. "Inappropriate Use of Antibiotics and Its Associated Factors among Urban and Rural Communities of Bahir Dar City Administration, Northwest Ethiopia”. PLoS ONE 10 (2015): e0138179.

27. Centre for Disease Dynamics, Economics and Policy (CDDEP). Global Antibiotic Resistance Partnership - Centre for Disease Dynamics, Economics and Policy (CDDEP) (2017). 
28. Lopes-Júnior., et al. "Decrease in Penicillin Sales in Brazil after Over-the-Counter Restrictions". Antimicrobial Agents and Chemotherapy 59 (2015): 5862-5863.

29. Ghafur A. About Us - Chennai Declaration (2013).

30. Huttner B., et al. "Characteristics and outcomes of public campaigns aimed at improving the use of antibiotics in outpatients in high-income countries". The Lancet 10 (2010): 17-31.

31. Johnson T., et al. "Health professions and the state in Europe" (2005).

32. Almaaytah A., et al. "Dispensing of non-prescribed antibiotics in Jordan. Patient Preference and Adherence (2015): 1389.

33. Morgan Daniel J., et al. "Non-Prescription Antimicrobial Use Worldwide: A Systematic Review". The Lancet infectious diseases 11.9 (2011): 692-701.

34. Both L., et al. "Analysis of licensed over-the-counter (OTC) antibiotics in the European Union and Norway, 2012". Eurosurveillance 20 (2014).

35. Ocan M., et al. "Patterns and Predictors of Self-Medication in Northern Uganda". PLoS ONE 9 (2014): e92323.

36. Olayemi O., et al. "Evaluation of Antibiotic Self-Medication Pattern amongst Undergraduate Students of Ahmadu Bello University (Main Campus), Zaria". Research Journal of Applied Sciences Engineering and Technology 2 (2010).

37. Bilal M. "Self-Medication with Antibiotics among People Dwelling in Rural Areas of Sindh". Journal of clinical and diagnostic research (2016).

38. Ramay BM., et al. "Comparing antibiotic self-medication in two socio-economic groups in Guatemala City: a descriptive cross-sectional study". BMC Pharmacology and Toxicology 16 (2015): 11.

39. Nahman C. "Antibiotics Without a Prescription?” (2014).

40. Markel Howard. "No Prescription for Antibiotics? No Problem". The New York Times, The New York Times (2002).

41. Abasaeed A., et al. "Self-medication with antibiotics by the community of Abu Dhabi Emirate, United Arab Emirates". The Journal of Infection in Developing Countries 3 (2009).
42. Awad AI and Aboud EA. Knowledge, Attitude and Practice towards Antibiotic Use among the Public in Kuwait". PLOS ONE 10 (2015): e0117910.

43. Shehnaz SI., et al. "Self-medication and related health complaints among expatriate high school students in the United Arab Emirates". Pharmacy Practice 11 (2013): 211-218.

44. Jamhour Antoun., et al. "Antibiotic knowledge and self-medication practices in a developing country: A cross-sectional study". American Journal of Infection Control 45 (2017): 384388.

45. Al-Azzam., et al. "Self-Medication with Antibiotics in Jordanian Population". International Journal of Occupational Medicine and Environmental Health 20 (2007): 373-380.

46. Llor C and Cots J. "The Sale of Antibiotics without Prescription in Pharmacies in Catalonia, Spain". Clinical Infectious Diseases 48 (2009): 1345-1349.

47. Widayati A., et al. "Self-medication with antibiotics in Yogyakarta City Indonesia: a cross sectional population-based survey". BMC Research Notes 4 (2011): 491.

\section{Volume 3 Issue 9 September 2019} (C) All rights are reserved by Carmen Aceijas and Dianna Henry Selvaraj. 